\title{
ENSAYO SOBRE LA IMPORTANCIA DE LA EDUCACIÓN SEXUAL EN PERSONAS CON DÉFICIT INTELECTUAL: REFLEXIONES DESDE LA TERAPIA OCUPACIONAL ${ }^{1}$
}

\author{
ESSAY ON THE IMPORTANCE OF SEX EDUCATION IN PEOPLE WITH INTELLECTUAL DEFICIT: \\ REFLECTIONS FROM OCCUPATIONAL THERAPY
}

\author{
Javiera Mella V. ${ }^{2}$
}

\section{Resumen}

En el presente ensayo se analiza y reflexiona sobre la importancia de la educación sexual en personas con déficit intelectual desde la Terapia Ocupacional, ya que sólo cuando se valida y justifica el por qué y para qué, se puede planificar el cómo y dónde intervenir. Para ello, se consideran las particularidades de este grupo y se analizan, desde el dominio de la Terapia Ocupacional (AOTA, 2008), el concepto de sexualidad formulado por la Organización Mundial de la Salud - OMS (2006), la necesidad y el impacto en sus procesos de integración social, así como algunas reflexiones bioéticas en torno al tema.

Bajo esta lógica de análisis teórico y reflexivo, es que surge la idea de considerar que la integración social implica la necesidad de maximizar la independencia y autonomía de la persona con déficit intelectual, también en su dimensión sexual, cuya vivencia y expresión se realiza cotidianamente. Por tanto, su educación deber ser una labor centrada en la persona y realizada de manera conjunta con su familia y el equipo multidisciplinario. En base a esto último, se concluye que no es necesario ser un experto en el tema para ofrecer la asesoría necesaria, por lo que los Terapeutas Ocupacionales deben formar parte de estos equipos, ya que poseen las herramientas necesarias.

\section{Palabras Clave}

Personas con déficit intelectual, educación sexual, Terapia Ocupacional.

\footnotetext{
${ }^{1}$ Este trabajo corresponde a la reflexión, en formato de ensayo, presentada para optar al título de Terapeuta Ocupacional, aprobado con distinción máxima (2012).

2 Terapeuta Ocupacional, Licenciada en Ciencias de la Ocupación Humana, Universidad de Chile.

Escuela Diferencial E-791, San Bernardo - Centro de Estimulación, desarrollo y terapias de rehabilitación.

Contacto: javieramellavenegas@gmail.com
} 


\begin{abstract}
The following essay analyzes and reflects on the importance of sex education in people with intellectual deficit from Occupational Therapy, as only when the why and the what for are validated and justified, it is possible to plan on how and where to intervene. In order to carry it out, the peculiarities of this group are considered as well as analyzed from the Occupational Therapy domain (AOTA, 2008), the concept of sexuality formulated by the World Health Organization - WHO (2006), the need and impact in their social integration processes as well as some bioethical reflections on the subject matter.

Under this reflective and theoretical logic analysis it emerges the idea of considering that social integration implies the need of maximizing the independence and autonomy of persons with intellectual deficit as well as their sexual dimension which expression and experience are daily performed. Therefore, his/her education must be a person-centered work and it also must be accomplished together with his/her family and the multidisciplinary team. Based on the latter, it is concluded that it is not necessary being an expert in the field to provide the essential advice, and then occupational therapists should be part of these teams, as they have the necessary tools.
\end{abstract}

\title{
Key Words
}

People with intellectual deficit, sex education, Occupational Therapy. 


\section{INTRODUCCIÓN}

Durante los últimos años, ha habido considerables avances en las intervenciones de las personas con déficit intelectual ( $P C D I)$. Mayoritariamente se reconocen los servicios de índole física, psicosocial, educativa y laboral, con el propósito de que puedan integrarse y desempeñarse en la sociedad, de la manera más independiente posible, evidenciando poco avance en la dimensión sexual de la PcDI (Rivera, 2008), no tan solo en el plano asistencial, sino que también educativo y familiar.

A pesar de la naturalidad de la sexualidad humana, su falta de consideración en este grupo podría estar sustentada en la existencia de prejuicios y/o erróneas creencias en relación a la sexualidad, más aun cuando se trata en personas con discapacidad. En el caso de las personas con déficit intelectual, al recibir tal etiqueta, en principio por la disminución en su capacidad cognitiva y adaptativa, reciben el mismo nivel de déficit en lo sexual y afectivo (Clemente, 2000), sin embargo esto es discapacitar doblemente y negar los derechos sexuales básicos.

La amplia gama de publicaciones en torno al tema, en su mayoría internacional, posee una visión crítica y defensiva de la educación sexual que debiesen tener las PcDI. Para ello utiliza un lenguaje con conceptos cercanos al dominio y quehacer de la Terapia Ocupacional (TO), aunque la minoría proviene desde la disciplina y que, por lo tanto, su enfoque y rol aun parecieran estar poco explorados y definidos. Así, cabe preguntarse ¿Cuál es la importancia que, desde la disciplina, se puede dar a la educación sexual de esta población?. Resuelta esta interrogante, se puede fijar el dónde y cómo intervenir, pues una estrategia básica para la promoción de la salud sexual ${ }^{3}$, es la validación y valoración de las intervenciones en el área (Couldrick, 2005).

Por esto, mediante el diálogo entre la literatura existente y un análisis personal, se realiza un acercamiento a la importancia de la educación sexual en dicho colectivo, reconociendo que, debido a sus características particulares, las PCDI requieren de una preparación específica en distintas áreas, con el fin de optimizar su autonomía e independencia y facilitar sus procesos de plena integración, lo que implica considerar su sexualidad; concepto analizado desde el Dominio de la Terapia Ocupacional (AOTA, 2008). Finalmente, se exponen algunas reflexiones bioéticas, las cuales nos llevan a revisar los Derechos Humanos y Sexuales, así como el deber profesional del Terapeuta Ocupacional, quien posee herramientas para ofrecer asesoría en sexualidad (Couldrick, 1998).

\footnotetext{
${ }^{3}$ Estado de bienestar físico, emocional, mental y social relacionado con la sexualidad (OMS, 2006).
} 


\section{DESARROLLO TEMÁTICO}

\section{PERSONAS CON DÉFICIT INTELECTUAL}

¿Quiénes son las personas con déficit intelectual?

En el 2002 la Asociación Americana de Discapacidad Intelectual y del Desarrollo (AAIDD) establece que el retraso mental es "una discapacidad caracterizada por limitaciones significativas en el funcionamiento intelectual y la conducta adaptativa tal como se ha manifestado en habilidades prácticas, sociales y conceptuales. Comienza antes de los 18 años" (en Ministerio de Educación - MINEDUC, 2007, p. 17).

Dicha definición se aleja del paradigma tradicional que define la discapacidad como un rasgo del individuo y se basa en la interacción de la persona y su contexto, tal como lo hace la Clasificación Internacional del Funcionamiento de la Discapacidad y de la Salud (OMS \& OPS, 2001). Sin embargo, y como lo plantea la CIF, existe un nuevo concepto de discapacidad $^{4}$, que lo diferencia de déficit o condición de salud. Su distinción hace emerger la interrogante de ¿Por qué denominar siempre y a todos como personas con discapacidad intelectual?, puesto que no podemos establecer con certeza que todos, producto de su déficit intelectual, poseen limitaciones y restricciones en todas las situaciones de participación en sus vidas cotidianas. Por lo tanto, se acepta la definición de la AAIDD, sin embargo no las palabras de discapacidad, ni de retraso mental, ya que este ensayo parte del supuesto que hay PCDI que pueden estar interesadas en demandar e iniciar procesos educativos (MINEDUC, 2007), incluso en sexualidad.

¿Qué necesidades poseen?

Los sistemas de clasificación de la AAIDD y de la Asociación Americana de Psiquiatría - APA (1995) incluyen los mismos tres criterios para el diagnóstico de déficit intelectual (o bien, retraso mental en su lenguaje). Esto es: Capacidad o funcionamiento intelectual significativamente inferior al promedio, limitaciones de las habilidades adaptativas e inicio anterior a los 18 años de edad. La APA (1995) define la capacidad intelectual por el coeficiente de inteligencia $(\mathrm{Cl})$, mientras que la capacidad adaptativa se refiere a la forma de afrontar efectiva y autónomamente las exigencias de la vida cotidiana.

\footnotetext{
${ }^{4}$ Se considera un término genérico que engloba las: deficiencias de las funciones y/o estructuras corporales, limitaciones en la actividad y restricciones en la participación; aludiendo a la interacción negativa entre un individuo y su contexto (OMS \& OPS, 2001).
} 
Ambas asociaciones consideran que la capacidad adaptativa de cada individuo puede estar influida por distintos factores (nivel de escolaridad, grado de déficit intelectual, motivación, oportunidades sociales y laborales, etc.). Pero además, que las PcDI "suelen presentar incapacidades adaptativas más que un Cl bajo" (APA, 1995, p. 42). De esto podemos desprender que las PcDI requieren apoyos y ayudas terapéuticas necesarias para mejorar su funcionamiento adaptativo e integrarse a su vida cotidiana. De hecho, Pello Yaben (1993) señala que las "dificultades adaptativas de estas personas son más el efecto de una educación deficiente que las consecuencias real de su propia deficiencia" (en García, 2003, p. 225).

Por lo tanto, aunque estas personas, como establecen Kempton et al. (1978), poseen dificultades para aprender por sus propios medios (en García, 2003), así como un nivel de conocimientos, por ejemplo en sexualidad, que tiende a ser más bajo que el resto de personas con algún tipo de déficit (Luengo, M.X. et al., 2000; Rueda \& Miranda, 2002), pueden beneficiarse de la educación y desarrollar habilidades adaptativas que les permitan desempeñarse en su vida cotidiana. La única distinción es que poseen un desarrollo evolutivo enlentecido y que requieren distintos grado de instrucción y asistencia, según el grado de déficit intelectual (APA, 1995).

Por otro lado, si bien no existen características comportamentales o de personalidad específicas que estén asociadas unívocamente al déficit intelectual (APA, 1995), hay quienes tildan de anormal lo pasivos, plácidos, dependientes, impulsivos o agresivos que puedan ser. No obstante estas conductas son normales y no justifican los mitos y prejuicios engendrados para este grupo; los cuales establecen que son personas asexuadas, incapaces de ser atractivas para otras, sin interés erótico, o que al despertar su sexualidad, lo hacen de manera salvaje y desbocada (López, 2002 en Insa, 2005; MINEDUC, 2007).

A pesar de ello, lamentablemente esto ha transformado su sexualidad en un ámbito plagado de prohibiciones, aunque como todos los individuos, las personas con déficit intelectual "poseen un cuerpo sexuado que experimenta: sensaciones, emociones, deseos, erotismo y placer; y que se relaciona con otros construyendo en el transcurso de su vida, su identidad como hombre o mujer" (MINEDUC, 2007, p. 10). Y aunque pueden tener un desarrollo sexual más lento (Clemente, 2000; García, 2003; MINEDUC, 2007; Rivera, 2008), no son niños en cuerpo de adultos y pueden experimentar una vida sexual satisfactoria, que sin duda tendrá su especificidad considerando sus posibilidades y limitaciones en capacidad intelectual y adaptativa. En esto, es necesario insistir que la presencia de un déficit intelectual, no implica un déficit sexual y menos una negación a una característica inherente del ser humano. 


\section{CONCEPTO DE SEXUALIDAD}

¿Qué es la Sexualidad?

La sexualidad es un aspecto central del ser humano, presente a lo largo de su vida. Abarca al sexo, las identidades y roles de género, el erotismo, el placer, la intimidad, la reproducción y la orientación sexual. Se vivencia y se expresa a través de pensamientos, fantasías, deseos, creencias, actitudes, valores, conductas, prácticas, papeles y relaciones interpersonales. La sexualidad puede incluir todas estas dimensiones, no obstante, no todas ellas se vivencian o se expresan siempre (OMS, 2006, p. 10).

A partir de esta definición, se interpreta que la sexualidad es esencial, constitutiva e inherente a todos los humanos, independiente de su condición física o mental. Toda persona vive en un cuerpo sexuado, por lo que su sexualidad se vivencia y expresa desde que está en el útero y va a la tumba. No sólo implica la relación sexual, como comúnmente se piensa, sino que también involucra las dimensiones afectivas, emocionales, biológicas, psicológicas, sociales, culturales, éticas, legales, históricas y espirituales (OMS, 2006), presentes de manera diferenciada en todo el ciclo vital de la persona y que la hacen desempeñarse y relacionarse con una determinada identidad.

Sexualidad: Consideraciones desde el Dominio de la Terapia Ocupacional

- Áreas de ocupación: La AOTA, mediante el Marco de Trabajo de la Terapia Ocupacional (2008), categoriza la participación en actividades sexualmente placenteras en las actividades básicas de la vida diaria (AVD-B), sin embargo ya se ha señalado que la sexualidad humana no solo es actividad sexual. La identidad y los roles de género se desarrollan, vivencian y expresan, a lo largo de la vida, mediante el desempeño en distintas actividades (AVD, tiempo libre, juegos y participación social, incluso en ambientes productivos) y no solo por el acto sexual propiamente tal. Además, la dimensión afectiva y cultural de la sexualidad, nos hace considerar las actividades como ocupaciones, las cuales involucran solo "aquellas actividades con sentido en que la persona participa cotidianamente y que puede ser nombrada por la cultura" (Álvarez et al., 2007, p. 78).

- Factores del cliente: Los valores, creencias y espiritualidad que posee cada persona influyen y son influidos por la motivación, actitudes, conductas y prácticas relacionadas a la vivencia y expresión de la sexualidad. Esta también posee una dimensión biológica que involucra estructuras y funciones corporales. Entre éstas últimas existen una variedad de textos que explican la multiplicidad de efectos y cambios en los distintos sistemas 
corporales. A modo general, funciones involucradas son las: mentales, sensoriales, neuromúsculo-esqueléticas, cardiovasculares, endocrinas, respiratorias, genito-urinarias y reproductivas. Si bien estas son importantes, la presencia o ausencia de estructuras o funciones corporales específicas, por ejemplo cognitivas, no necesariamente asegura el éxito o dificultad en el desempeño (AOTA, 2008).

- Habilidades de desempeño: La sexualidad, al vivenciarse y expresarse mediante distintas ocupaciones, requiere de la adquisición y práctica de acciones observables, concretas y dirigidas (Fisher, 2006 en AOTA, 2008) que poseen las habilidades motoras, sensorioperceptuales, cognitivas, de regulación emocional y de comunicación e interacción, de manera específica en cada etapa del ciclo vital.

- Patrones de desempeño: Los hábitos y rutinas son especialmente necesarios, por ejemplo, en las actividades de higiene relacionadas a la sexualidad. Mientras que las relaciones interpersonales, fantasías, deseos, actitudes, creencias, valores, conductas y prácticas que posee cada persona en la vivencia y expresión de su sexualidad (OMS, 2006), guardan una estrecha relación con su identidad y roles de género. Estos últimos se desarrollan y expresan en las ocupaciones, cumpliendo con un conjunto de normas conceptualizadas y definidas por la persona e influenciadas por su cultura. Por otro lado, la sexualidad también involucra el ejercicio de rituales, o bien, "acciones simbólicas con significado espiritual, cultural o social (...), poseen un fuerte componente afectivo (...)" (Fiese et al., 2002; Segal, 2004, en AOTA, 2008, p. 642).

- Contexto y medioambiente: La sexualidad ocurre en un medioambiente físico, social y personal, con el cual genera una interdependencia (AOTA, 2008), en cuanto afecta la accesibilidad e influye en la calidad del desempeño. Así mismo, el contexto cultural y temporal a menudo influye en cómo la sexualidad es vivenciada y expresada.

- Demandas de la actividad: Como se ha establecido, la sexualidad se vivencia y expresa en distintas ocupaciones, por lo que posee una diversidad de demandas, en cuanto a objetos, espacios, secuencias, relaciones y acciones (AOTA, 2008) que éstas puedan requerir. Como sea, se debe tener presente las potencialidades y limitaciones que la PcDI posee, descritas anteriormente. 


\section{IMPORTANCIA DE LA EDUCACIÓN SEXUAL EN PERSONAS CON DÉFICIT INTELECTUAL: REFLEXIONES DESDE LA TERAPIA OCUPACIONAL.}

La palabra educación proviene del latín educere: guiar, conducir o educare: formar, instruir $y$, como tal, se reconoce como un proceso en el cual se transmiten conocimientos. Sin embargo, también involucra aptitudes, valores, costumbres y formas de actuar, necesarias a lo largo de toda la vida (Azaustre, 2009). De hecho, la UNESCO establece que la educación debe cimentarse en el aprender a conocer, hacer, ser y convivir (Delors, 1997). Al permitir el desarrollo individual, mediante la asimilación de una cultura socialmente organizada, la educación es un bien humano que surge de la necesidad de desarrollarse como tal y ante la que toda persona tiene derecho (MINEDUC, 2004).

Las PcDI pueden, deben y son integradas socialmente en la medida que poseen la capacidad, el interés y la equiparación de oportunidades (Grupo Latinoamericano de Rehabilitación Profesional en SENADIS, s.f.), ya que ante sus características cognitivas y adaptativas particulares, necesitan de una preparación específica con el fin de optimizar su autonomía e independencia. Así surge el concepto de Transición a la Vida Adulta (TVA), el cual nos habla de un proceso dinámico de transformación y preparación de competencias individuales necesarias para la vida adulta y la consecución de un proyecto de vida en un mundo social. En dicho proceso existe una interrelación entre factores personales y contextuales en todos los ciclos vitales del desarrollo humano, por lo que ha sido necesario formular ayudas terapéuticas y/o estrategias orientadas a maximizar las potencialidades y equiparar las oportunidades de las PcDI, mediante la generación de los denominados Apoyos para la Transición a la Vida Adulta (MINEDUC \& Universidad Católica Silva Henríquez UCSH, 2010).

Esta orientación de la TVA en las PCDI nos hace reflexionar sobre los apoyos, oportunidades e intervenciones que, como sociedad y/o Terapeutas Ocupacionales, estamos ofreciendo para que se realicen como seres humanos integrados socialmente. ¿Estamos siendo realmente integrales? En ese sentido, es necesario destacar que los objetivos de intervención "no deben estar dirigidos únicamente al empleo. Es necesario que abarquen todas las dimensiones relacionadas con un adecuado ajuste a la vida adulta y una integración a la sociedad" (Carulla \& Rodríguez, 2001 en Ramírez, 2005, p. 85). Por lo tanto, no se puede hablar de integración plena, ni de apoyos para la TVA, si no es considerada su sexualidad, la cual es un aspecto central para "acceder a la madurez y lograr la realización personal y social, anhelada por todos los seres humanos" (Tyne, 2003 en Ramírez, 2005, p.92). 
Por lo tanto, educación e integración son conceptos y procesos altamente relacionados en esta población, más aun si el tema central es la sexualidad, y cuando se considera que la PcDI es más vulnerable, sobretodo en una sociedad donde lo sexual ocupa un lugar importante (García, 2003). Las PCDI dependen de los apoyos constantes que se entreguen a lo largo de su vida y en todas sus dimensiones, para desarrollar una sexualidad sana, adecuada y plena. Mediante la educación que ofrezca la TO, se pueden atender a las maneras de comportarse en el hacer, pensar y sentir de la PcDI, lo cual le permitirá gozar de bienestar biopsicosocial y desarrollar herramientas acorde a sus factores personales y la demanda de cada actividad relacionada a lo sexual, mediante la integración de las habilidades de desempeño en ocupaciones que le permita cumplir con sus roles, así como identificar y reafirmar sus valores, creencias e identidad, al facilitar la vivencia y expresión autónoma, independiente y cotidiana de su sexualidad.

Por otro lado, ante la no capacitación en materia sexual, no es justo, ni ético esperar un comportamiento social adecuado en las PcDI (García, 2003). Además, también podría aumentar su invalidez (Rueda, 2003), por cuanto se limitan actividades y se restringe su participación; Aumentan los riegos asociados a la actividad sexual, tales como embarazo no deseado o abusos (Insa, 2005), más aun cuando se considera que la PcDI es más vulnerable. Por otro lado, no ofrecer educación sexual, es negar la equiparación de oportunidades que permitan ejercer su derecho de explorar y satisfacer sus intereses $y / 0$ necesidades sexuales. Por ello, los prejuicios que existen en torno a su sexualidad no deben ser aceptados, y las leyes de esta índole deben ser aplicadas y promovidas con el fin de proteger su dignidad humana (Rueda, 2003).

Bajo la misma línea de reflexiones en torno a lo bioético, y por ejemplo en el ámbito de los métodos anticonceptivos y las relaciones sexuales, se propende a considerar a la PCDI como falta de capacidad para prestar adecuadamente su consentimiento. Si bien es cierto, que no podemos saber con certeza las posibilidades y limitaciones de autonomía generales de este grupo, podemos establecer que al disminuir su déficit y en condiciones facilitadoras, es decir ayudándole a entender con una completa y adecuada información adaptada (Kempton, 1985 en García, 2003; Rueda \& Miranda, 2002), un no menor porcentaje puede y debe ser partícipe de la toma de decisiones, en la medida de lo posible. Por lo tanto, una educación sexual adecuada salvaguarda su autonomía, integridad y salud sexual, así como su derecho de ser educado (Rueda, 2003), pues "cada individuo, no interesa cuál sea su discapacidad, tiene derecho al libre acceso a la información necesaria sobre la sexualidad y, lo que es más importante, cada persona tiene derecho a la expresión de su sexualidad" (Gordon, 1981 en Rueda \& Miranda, 2002, p. 134). Es preciso mencionar que este extracto de la Declaración de los Derechos Sexuales de los discapacitados (1981) es congruente con 
la Declaración Universal de los Derechos Sexuales (1999), los cuales son considerados "derechos humanos fundamentales y universales" (Asociación Mundial de Sexología, 1999).

Por lo tanto, como establecen distintos Terapeutas Ocupacionales dedicados a la asesoría sexual (Couldrick, 2005; Friedman, 1997; McKenna, 2005), dentro del rol profesional se debe promover la salud sexual, en congruencia con valores de la profesión en su compromiso con el bienestar biopsicosocial (beneficencia), la evitación de daños (no maleficencia), así como el respeto de las creencias, deseos y derechos de los usuarios (autonomía y justicia) (AOTA, 1998). Por lo tanto, como Terapeutas Ocupacionales, valoremos y reconozcamos la sexualidad de las PCDI para "velar por los intereses y derechos de quienes requieran nuestros servicios" (Colegio de Terapeutas Ocupacionales de Chile A.G, s.f., p. 1), mediante una adecuada y oportuna educación sexual.

\section{CONCLUSIÓN}

A pesar de las necesidades sexuales y educativas propias de las PCDI, su educación sexual pareciera ser aun un tema pendiente; una problemática llena de prejuicios y creencias erróneas, y por ello desatendida incluso en lo que respecta a la TO (Couldrick, 2005; Friedman, 1997; MacKenna, 2005).

Aunque la TO pareciera tener aún un enfoque y rol poco definido, se ha llegado a establecer que la sexualidad es un elemento constitutivo de todo ser humano y que posee una participación y consideración en cada área del dominio de la TO; Por cuanto, incluso en la PcDI, ésta se vivencia y expresa cotidianamente, en contextos determinados y acorde a los factores personales y la demanda de cada actividad relacionada a lo sexual, mediante la integración de las habilidades en el desempeño y una serie de ocupaciones (y no tan solo una AVD) que permiten cumplir con los roles percibidos y reafirmar los valores, creencias e identidad de la PcDI. Por ello, en TO se debe ser proactivo frente a la dimensión sexual del usuario (Friedman, 1997), porque este colectivo lo necesita, debido a sus características particulares, para madurar y realizarse como una persona sexual, con el fin de integrarse de manera plena y segura en la sociedad. Somos los Terapeutas Ocupacionales los que buscamos el mayor grado de autonomía e independencia posible, resguardando los intereses y derechos de las personas, por lo que la educación sexual puede y debe ser un eficaz y necesario Apoyo para las intervenciones en la TVA de la PcDI.

Por lo tanto, la integralidad y relevancia de la educación sexual hace de ésta una labor durante todo el ciclo vital y conjunta con la persona, su familia y el equipo multidisciplinario. No se debe ser un experto para educar estos temas (García, 2003; 
MINEDUC, 2007), sino que basta con estar abierto al diálogo y tener una actitud positiva frente a la sexualidad, aunque esto no nos libera de la necesidad de educarnos en elementos teóricos y técnicos cuando sea necesario, pues aunque existan publicaciones que intentan coordinar la respuesta educativa en sexualidad para este colectivo, éstas no pueden suplir la formación profesional.

\section{REFERENCIAS BIBLIOGRÁFICAS.}

Álvarez, E., Gómez, S., Muñoz, I., Navarrete, E., Riveros, M.E., Rueda, L., Salgado, P., Sepúlveda, R. \& Valdebenito, A. (2007). Definición y desarrollo del concepto de Ocupación: ensayo sobre la experiencia de construcción teórica desde una identidad local. Revista Chilena de Terapia Ocupacional, 7, 76-82.

Asociación Americana de Psiquiatría - APA. (1995). DSM-IV. Manual diagnóstico y estadístico de los trastornos mentales (4a. Ed.). Barcelona: Masson.

Asociación Americana de Terapia Ocupacional - AOTA. (1998). Código ético de la Terapia Ocupacional. Revista Americana de Terapia Ocupacional (AJOT), 52, 881-884.

Asociación Americana de Terapia Ocupacional - AOTA. (2008). Marco de Trabajo para la Práctica de la Terapia Ocupacional: dominio y proceso (2a . Ed.). Revista Americana de Terapia Ocupacional (AJOT), 62 (6), 625-663.

Asociación Mundial de Sexología. (1999). Declaración de los Derechos Sexuales. $14^{\circ}$ Congreso Mundial de Sexología, Asamblea General de la Asociación Mundial de Sexología. Hong Kong: Author.

Azaustre, A. (2009). La educación. Revista digital enfoques educativos, 50, 21-30. Recuperado el 03/01/2012, de http://www.enfoqueseducativos.es/enfoques/enfoques_50.pdf\#page=21

Clemente, A. (2000). Desarrollo de la sexualidad del discapacitado psíquico. En Amor, J.R. (Ed.). Sexualidad y personas con discapacidad psíquicas, p. 69-83. Madrid: FEAPS. Recuperado el 06/01/2012, http://www.feaps.org/biblioteca/sexualidad_ydi/04_desarrollosexual.pdf Colegio de Terapeutas Ocupacionales de Chile A.G. (s.f.). Código de Ética Profesional. A.G. Recuperado el 26/12/2011, de http://www.terapiaocupacional.cl/documentos/codigo\%20de\%20etica.php 
Couldrick, L. (1998). Sexual Issues: an area of concern for Occupational Therapists?. British Journal of Occupational Therapy, 61 (11), 493-496.

Couldrick, L. (2005). Sexual Expression and Occupational Therapy. British Journal of Occupational Therapy, 68 (7), 315-318.

Delors, J. (1997). La Educación: encierra un tesoro; informe a la UNESCO de la Comisión Internacional sobre la Educación para el Siglo XXI (2ª . Ed.). México: Ediciones UNESCO.

Friedman, J.D. (1997). Sexual expression, the forgotten component of ADL, OT Practice, 20-25.

García, J. L. (2003). Educación sexual y afectiva en personas con discapacidad mental. Santiago de Chile: Fundación HINENI Chile.

Insa, E. (2005). El desarrollo de la sexualidad en la deficiencia mental. Revista Interuniversitaria de Formación de Profesorado, 19 (3), 327-343. Recuperado el 21/12/2011, de http://redalyc.uaemex.mx/pdf/274/27411927015.pdf

Luengo, M.X., Toledo, V., Fuentes, M.E., Lobos, L., Molina, R. \& Molina, T. (2000). Adolescentes discapacitados: talleres de afectividad y sexualidad, Revista Chilena de Pediatría, 71 (1), 24-31. Recuperado el 27/12/2011, de http://www.scielo.cl/scielo.php?script=sci_arttext\&pid=S0370$41062000000100006 \&$ lng=es\&nrm=iso.

McKenna, K. (2005). Sexualidad y discapacidad. En Crepeau, E., Cohn, E. \& Schell, B. (Eds.). Willard \& Spackmann. Terapia Ocupacional (10a. Ed.), p. 541-546. Madrid: Editorial Médica Panamericana.

Ministerio de Educación - MINEDUC. (2004). Nueva perspectiva y visión de la educación especial. Informe de la comisión de expertos. Santiago de Chile: K-Diseño. Recuperado el 06/01/2012,

http://www.mineduc.cl/usuarios/edu.especial/File/DOCUMENTOS\%20VARIOS\%202008/Infor meComisiOnExpertos.pdf

Ministerio de Educación - MINEDUC. (2007). Guía para el docente: Educación sexual de niños, niñas y jóvenes con discapacidad intelectual. Santiago de Chile: Author. 
Ministerio de Educación - MINEDUC \& Universidad Católica Silva Henríquez - UCSH. (2010). Jornada de Capacitación. Fase presencial. Documentos de lectura. "Elaboración, ejecución, seguimiento y evaluación de propuesta de estrategias innovadoras de apoyo a la transición para la Vida Adulta de los estudiantes que presentan Necesidades Educativas Especiales asociadas a la Discapacidad Intelectual". Santiago de Chile: Author.

Organización Mundial de la Salud - OMS. (2006). Defining sexual health: report of a technical consultation on sexual health. Recuperado el 26/12/2011, de http://www.who.int/reproductivehealth/publications/sexual_health/defining_sexual_healt h.pdf

Organización Mundial de la Salud - OMS \& Organización Panamericana de la Salud - OPS. (2001). Clasificación Internacional del Funcionamiento de la Discapacidad y de la Salud. Madrid: Author.

Ramírez, D. (2005). En busca de un estilo de vida más inclusivo para las personas adultas con discapacidad intelectual, Revista Educación y Pedagogía, 17 (41), 85-94. Recuperado el 04/01/2012, de http://aprendeenlinea.udea.edu.co/revistas/index.php/revistaeyp/article/viewFile/6030/ 5436

Rivera, P. (2008). Sexualidad de los niños, niñas y jóvenes con discapacidad. Revista educación, 32 (1), 157-170. Recuperado el 15/12/2011, de http://redalyc.uaemex.mx/pdf/440/44032111.pdf

Rueda, L. (2003). Aspectos éticos de la sexualidad en los discapacitados. Recuperado el 22/12/2011, de http://consejoderedieiupn.blogspot.com/2005/08/aspectos-ticos-de-lasexualidad-en-los.html

Rueda, L. \& Miranda, O. (2002). Principales dilemas bioéticos en las personas con discapacidad prolongada, Acta Bioethica, 8 (1), 127-135. Recuperado el 14/12/2011, de http://www.scielo.cl/scielo.php?script=sci_arttext\&pid=S1726-

569X2002000100012\&lng=es\&nrm=iso

Servicio Nacional de la Discapacidad - SENADIS. (s.f.). Glosario. Recuperado el 03/01/2012, de http://www.senadis.gob.cl/glosario/index.php 